\title{
INFINITE DIMENSIONAL STATIONARY SEQUENCES WITH MULTIPLICITY ONE
}

\author{
A. MAKAGON and H. SALEHI
}

\begin{abstract}
It is shown that an infinite dimensional stationary sequence is deterministic if and only if some related family of stationary sequences with multiplicity one has this property. A spectral characterization for regular processes with multiplicity one is discussed. The relation between factorability and the conjugate analyticity of the range of the spectral density is examined. An example of a factorable spectral density without conjugate analytic range is given.
\end{abstract}

\section{Introduction}

In [7] Niemi has observed that a multivariate stationary sequence is deterministic if and only if some related family of stationary sequences with multiplicity one has this property. The purpose of this paper is twofold. First we extend Niemi's result to the infinite dimensional case. Our approach to the problem is through spectral multiplicity theory which is applicable to both the finite and infinite dimensional cases. The second aim is to give a spectral characterization for regular processes of multiplicity one. We summarize the content of the paper as follows. In Section 2 we state some known facts from the theory of spectral types. Section 3 contains the extension of Niemi's results. In Section 4, analytic characterizations for the regularity of a stationary sequence with multiplicity one are studied. These characterizations are of two types. One pertains to the notion of the conjugate analyticity of the range of the spectral density, as introduced by H. Helson in [5], and the behavior of the norm of the density. The relation between factorability and the conjugate analyticity of the range of the density is examined. An example of a factorable spectral density without conjugate analytic range is given. The other type deals with an extension of Matveev's results in [6] connecting the factorization problem to the Nevanlinna class of functions.

Throughout the paper, $\mathbf{N}, \mathbf{Z}$ and $\mathbf{C}$ stand for positive integers, all integers and complex numbers. $\mathscr{H}, \mathscr{K}$ will always denote complex Hilbert spaces with a

AMS 1980 Subject Classification: Primary 60G25; Secondary 60G10, 47A68.

Key words and phrases: infinite dimensional stationary sequence, multiplicity, determinism. conjugate analytic range, factorization of spectral density. 
norm $|\cdot|$, and an inner product $(\cdot, \cdot)$. By $L(\mathscr{H}, \mathscr{K})$ we will denote the space of all continuous linear operators from $\mathscr{H}$ into $\mathscr{K}$ equipped with the operator norm | |. If $\mathscr{H}=\mathscr{K}$, we will abbreviate $L(\mathscr{H}, \mathscr{H})$ as $L(\mathscr{H}) . L^{+}(\mathscr{H})$ will stand for the subset of $L(\mathscr{H})$ consisting of all selfadjoint operators $T \in L(\mathscr{H})$ such that $(T x, x) \geqq 0$ for all $x \in \mathscr{H}$. I will denote the identity operator. For $x$ and $y$ in $\mathscr{H}$, $x \otimes y$ is the operator in $L(\mathscr{H})$ defined by the formula $(x \otimes y) z=(z, y) x, z \in \mathscr{H}$. If $\mathscr{M}$ is a closed subspace of $\mathscr{H}$, then we will denote the orthogonal projection operator onto $\mathscr{M}$ by $P_{\mathscr{M}} . \mathscr{M}^{\perp}$ will stand for the orthogonal complement of $\mathscr{M}$ in $\mathscr{H}$. The symbol $\oplus$ indicates an orthogonal sum. The set of all linear combinations of elements of $A$ will be denoted by $\operatorname{sp} A$. If $T \in L(\mathscr{H}, \mathscr{K})$, then $T \mathscr{H}$ is the range of $T$, and $\overline{T \mathscr{H}}$ denotes the closure of the range of $T$. If $\Sigma$ is a $\sigma$-algebra of subsets of a set $\Omega$ and $\mu$ is a $\sigma$-additive $\sigma$-finite scalar measure on $\Sigma$, then we will denote the space of all $\mathscr{H}$-valued $p$-Bochner integrable functions on $\Omega$ by $L^{p}(\Omega, \Sigma, \mu, \mathscr{H})$. The Lebesgue measure on $(-\pi, \pi]$ is denoted by $d t$. If $\Omega=(-\pi, \pi], \Sigma$ is the Borel $\sigma$-algebra of measurable sets in $(-\pi, \pi]$ and $\mu=d t$, then we will simply write $L^{p}(\mathscr{H})$ instead of $L^{p}((-\pi, \pi], \Sigma, d t, \mathscr{H})$. The set of all functions $f \in L^{p}(\mathscr{H}), p \geqq 1$, such that $\int_{-\pi}^{\pi} e^{- \text {int }} f(t) d t=0$ for all $n \geqq 1(n \leqq-1)$, will be denoted by $L_{+}^{p}(\mathscr{H})\left(L_{-}^{p}(\mathscr{H})\right.$, respectively). The Hardy classes $H^{p}$ and the Nevanlinna class $N_{+}$can be found in [4]. The inequality $k<n+1$ means $k \leqq n$ if $n<\infty$, and $k<\infty$ if $n=\infty$. For two measures $\mu$ and $\nu$ we mean by $\mu \ll v$ that $\mu$ is absolutely continuous with respect to $v$.

\section{Preliminary results}

Let $\mathscr{K}$ be a Hilbert space and let $(\Omega, \Sigma)$ be a measurable space in the sense that $\Sigma$ is a $\sigma$-algebra of subsets of a set $\Omega$.

A function $E: \Sigma \rightarrow L(\mathscr{K})$ is said to be a spectral measure in $\mathscr{K}$ if

(i) for every $x, y \in \mathscr{K},(E(\cdot) x, y)$ is a countably additive set function on $\Sigma$,

(ii) for every $\Delta \in \Sigma, E(\Delta)$ is an orthogonal projection operator in $\mathscr{K}$,

(iii) $E(\Omega)=I$.

If $E$ is a spectral measure in $\mathscr{K}$ and $x \in \mathscr{K}$, then $\mathscr{M}(E, x)$ will denote the smallest closed subspace of $\mathscr{K}$ containing $x$ which is invariant under $E(\Delta), \Delta \in \Sigma$, i.e.,

$$
\mathscr{M}(E, x)=\overline{\mathrm{sp}}\{E(\Delta) x: \Delta \in \Sigma\} .
$$

The space $\mathscr{K}$ is said to be countably generated by $E$ if there exists a countable set $\left\{x_{k}: k=1,2, \ldots\right\} \subset \mathscr{K}$ such that $\overline{\mathrm{sp}}\left\{E(\Delta) x_{k}: \Delta \in \Sigma, k=1,2, \ldots\right\}=\mathscr{K}$.

2.1. Definition. Suppose that $\mathscr{K}$ is countably generated by a spectral measure $E$. The smallest number $n$ in $\mathrm{N} \cup\{+\infty\}$ for which there exists a set $\left\{x_{k}: k<n+1\right\} \subset \mathscr{K}$ with the property

$$
\mathscr{K}=\overline{\mathrm{sp}}\left\{E(\Delta) x_{k}: \Delta \in \Sigma, k<n+1\right\}
$$

is said to be the multiplicity of $E$ in $\mathscr{K}$, and is denoted by $m(E, \mathscr{K})$. 
The following lemma plays an important role in this paper, and its proof can be found in [3] p. 914-918.

2.2. Lemma. Suppose that $\mathscr{K}$ is countably generated by a spectral measure $E$.

(A) If $n=m(E, \mathscr{K})$, then there exist a decreasing sequence of set $\Delta_{k} \in \Sigma$ and a collection of vectors $x_{k} \in \mathscr{K},\left|x_{k}\right|=1,1 \leqq k<n+1$ such that

(i) $\mathscr{K}=\oplus_{k=1}^{n} \mathscr{M}\left(E, x_{k}\right)$,

(ii) $\left|E(\Delta) x_{k}\right|^{2}=\left|E\left(\Delta \cap \Delta_{k}\right) x_{1}\right|^{2}$ for all $\Delta \in \Sigma, \quad 1 \leqq k<n+1$,

(iii) $\frac{d\left|E(\cdot) x_{k}\right|^{2}}{d\left|E(\cdot) x_{1}\right|^{2}}=1 \quad\left|E(\cdot) x_{1}\right|^{2} \quad$ a.e. on $\quad \Delta_{k}, 1 \leqq k<n+1$.

(B) If $\mathscr{K}=\bigoplus_{k=1}^{n} \mathscr{M}\left(E ; x_{k}\right)=\bigoplus_{j=1}^{m} \mathscr{M}\left(E, y_{j}\right)$, where $m, n \in \mathbf{N} \cup\{+\infty\}, 0 \neq x_{k} \in \mathscr{K}$, $1 \leqq k<n+1,0 \neq y_{j} \in \mathscr{K}, 1 \leqq j<m+1$ such that

$$
\begin{aligned}
& \left|E(\cdot) x_{1}\right|^{2} \gg\left|E(\cdot) x_{2}\right|^{2} \gg \ldots \quad \text { and } \\
& \left|E(\cdot) y_{1}\right|^{2} \gg\left|E(\cdot) y_{2}\right|^{2} \gg \ldots,
\end{aligned}
$$

then $n=m=m(E, \mathscr{K})$ and moreover for every $k<n+1=m+1$. The measures $\left|E(\cdot) x_{k}\right|^{2}$ and $\left|E(\cdot) y_{k}\right|^{2}$ are equivalent.

Any sequence of measures $\mu_{k}(\cdot)=\left|E(\cdot) x_{k}\right|^{2}$ satisfying the condition in (B) constitutes the so-called spectral types of $E$ in $\mathscr{K}$. It follows from (B) that spectral types of $E$ in $\mathscr{K}$ are uniquely determined up to the equivalence of measures. Therefore we may speak of the spectral types of $E$, and call $\left|E(\cdot) x_{1}\right|^{2}$ the maximal spectral type of $E$ in $\mathscr{K}$.

2.3. Lemma. Suppose that $\mathscr{K}$ is countably generated by a spectral measure $E$. Let $\mathscr{M}$ be a closed subspace of $\mathscr{K}$ invariant under all $E(\Delta), \Delta \in \Sigma$.

(A) If the maximal spectral type of $E$ in $\mathscr{M}$ is singular with respect to the maximal spectral type of $E$ in $\mathscr{M}^{\perp}$, then

$$
m(E, \mathscr{K})=\max \left(m(E, \mathscr{M}), m\left(E, \mathscr{M}^{\perp}\right)\right) .
$$

(B) If the maximal spectral type of $E$ in $\mathscr{M}$ is absolutely continuous with respect to all the spectral types of $E$ in $\mathscr{M}^{\perp}$, then

$$
m(E, \mathscr{K})=m(E, \mathscr{M})+m\left(E, \mathscr{M}^{\perp}\right) .
$$

Proof. Suppose that $\mathscr{M}=\bigoplus_{i=1}^{n} \mathscr{M}\left(E, x_{i}\right)$ with $x_{i} \neq 0$, and

$$
\begin{aligned}
& \left|E(\cdot) x_{1}\right|^{2} \gg\left|E(\cdot) x_{2}\right|^{2} \gg \ldots \quad \text { and that } \mathscr{M}^{\perp}=\bigoplus_{i=1}^{m} \mathscr{M}\left(E, y_{i}\right), \quad y_{i} \neq 0 \\
& \left|E(\cdot) y_{1}\right|^{2} \gg\left|E(\cdot) y_{2}\right|^{2} \gg \ldots, \quad n, m \in \mathbf{N} \cup\{+\infty\} .
\end{aligned}
$$

(A) Let

$$
z_{i}=\left\{\begin{array}{lll}
x_{i}+y_{i} & \text { if } & 1 \leqq i<\min (m, n)+1 \\
x_{i} & \text { if } & m<n, \min (m, n)<i<\max (m, n)+1, \\
y_{i} & \text { if } & m>n, \min (m, n)<i<\max (m, n)+1
\end{array}\right.
$$


Since $\left|E(\cdot) x_{i}\right|^{2} \perp\left|E(\cdot) y_{i}\right|^{2}, 1 \leqq i<\min (n, m)+1$, there exist sets $\Delta_{i} \in \Sigma$ such that $\left|E\left(\Delta_{i}\right) x_{i}\right|^{2}=\left|E(\Omega) x_{i}\right|^{2}$ and $\left|E\left(\Delta_{i}^{c}\right) y_{i}\right|^{2}=\left|E(\Omega) y_{i}\right|^{2}$, where $\Delta^{c}$ denotes the complement of a set $\Delta$. It implies that $E\left(\Delta_{i}\right) z_{i}=x_{i}$ and $E\left(\Delta_{i}^{c}\right) z_{i}=y_{i}, 1 \leqq i<\min (n, m)+1$. Thus $\mathscr{M}\left(E, x_{i}\right) \oplus \mathscr{M}\left(E, y_{i}\right)=\mathscr{M}\left(E, z_{i}\right)$. Consequently, $\oplus_{i=1}^{k} \mathscr{M}\left(E, z_{l}\right)=\mathscr{K}$, where $k=\max (n, m)$. Since $\left|E(\cdot) z_{1}\right|^{2} \gg\left|E(\cdot) z_{2}\right|^{2} \gg \ldots$, it follows from Lemma 2.2 (B) that $m(E, \mathscr{K})=\max \left(m(E, \mathscr{M}), m\left(E, \mathscr{M}^{\perp}\right)\right)$.

(B) If $m\left(E, \mathscr{M}^{\perp}\right)=+\infty$, the statement is obvious. Suppose that $m\left(E, \mathscr{M}^{\perp}\right)=$ $m<\infty$ and define

$$
z_{i}= \begin{cases}y_{i}, & 1<i \leqq m, \\ x_{i-m}, & m<i<n+m+1 .\end{cases}
$$

Then $\mathscr{K}=\bigoplus_{i=1}^{m+n} \mathscr{M}\left(E, z_{i}\right)$ and $\left|E(\cdot) z_{1}\right|^{2} \gg\left|E(\cdot) z_{2}\right|^{2} \gg \ldots$ Thus, by Lemma 2.2 (B), $m(E, \mathscr{K})=m+n$.

2.4. Lemma. Let $\mathscr{H}$ be a separable Hilbert space and let $E(\Delta)$ denote the operator of multiplication by $1_{\Delta}$ in $L^{2}(\Omega, \Sigma, \mu ; \mathscr{H})$. Suppose that $\mathscr{K}$ is a countably generated subspace of $L^{2}(\Omega, \Sigma, \mu ; \mathscr{H})$. Let $f_{j}, j=1,2, \ldots$ be any family of elements in $\mathscr{K}$ such that $\mathscr{K}=\overline{\mathrm{sp}}\left\{E(\Delta) f_{j}: j=1,2, \ldots, \Delta \in \Sigma\right\}$. For every $\omega \in \Omega$ let us define

Then:

$$
\overline{\overline{\mathscr{K}}}(\omega)=\overline{\mathrm{sp}}\left\{f_{j}(\omega): j=1,2, \ldots\right\} .
$$

(i) $\overline{\overline{\mathscr{K}}}(\cdot)$ does not depend on the choice of $\left\{f_{j}: j=1,2, \ldots\right\}$ in the sense of $\mu$ almost everywhere equality.

(ii) $\mathscr{K}=\left\{f \in L^{2}(\Omega, \Sigma, m ; \mathscr{H}): f(\omega) \in \overline{\overline{\mathscr{K}}}(\omega) \mu\right.$ a.e. $\}$.

(iii) $m(E, \mathscr{K})=\mu$-ess $\sup (\operatorname{dim} \overline{\overline{\mathscr{K}}}(\cdot))$.

Proof. The proofs of both parts (i) and (ii) can be given by duplicating those of Rozanov's in [9], p. 89-91.

(iii). Let $n=m(E, \mathscr{K})$. From Lemma 2.2 (A) it follows that there exist $g_{k} \in \mathscr{K}$, $\left|g_{k}\right|=1$ and a decreasing family of sets $\Delta_{k} \in \Sigma, 1 \leqq k<n+1$, such that

$$
\begin{aligned}
(*) & \mathscr{K}=\oplus_{k=1}^{n} \mathscr{M}\left(E, g_{k}\right), \\
(* *) & \left|E\left(\Delta \cap \Delta_{k}\right) g_{1}\right|^{2}=\left|E(\Delta) g_{k}\right|^{2}, \quad 1 \leqq k<n+1, \\
(* * *) & \frac{d\left|E(\cdot) g_{k}\right|^{2}}{d\left|E(\cdot) g_{1}\right|^{2}}=1 \quad \mu \text { a.e. on } \Delta_{k}, \quad 1 \leqq k<n+1 .
\end{aligned}
$$

By (i), $\operatorname{dim} \overline{\overline{\mathscr{K}}}(\omega)=\operatorname{dim} \overline{\mathrm{sp}}\left\{g_{k}(\omega) ; 1 \leqq k<n+1\right\} \geqq n=m(E ; \mathscr{K}) \mu$ a.e. We shall prove that for every integer $k<n+1, \operatorname{dim} \overline{\overline{\mathscr{K}}}(\cdot) \geqq k$ on a set of positive measure $\mu$, which will complete the proof.

Let $\Delta_{0}=\left\{\omega:\left|g_{1}(\omega)\right| \neq 0\right\}$. Since $E(\Delta) g_{i} \perp E\left(\Delta^{\prime}\right) g_{j}$ for all $\Delta, \Delta^{\prime} \in \Sigma$ and $i \neq j$, $g_{i}(\omega) \perp g_{j}(\omega) \quad \mu$ a.e. for all $i \neq j$. Let $k<n+1$ be fixed. Since $\left|E\left(\Delta_{0} \cap \Delta_{k}\right) g_{j}\right|^{2}=$ $\left|E\left(\Delta_{k}\right) g_{1}\right|^{2}=\left|g_{k}\right|^{2} \neq 0, \mu\left(\Delta_{0} \cap \Delta_{k}\right) \neq 0$. Let $\Delta \subset \Delta_{0} \cap \Delta_{k}$. From (**) it follows that 
for every $1 \leqq j \leqq k, \int_{\Delta}\left|g_{j}\right|^{2} d \mu=\left|E(\Delta) g_{j}\right|^{2}=\left|E\left(\Delta \cap \Delta_{j}\right) g_{1}\right|^{2}=\left|E(\Delta) g_{1}\right|^{2}=\int_{\Delta}\left|g_{1}\right|^{2} d \mu \neq 0$, provided $\mu(\Delta) \neq 0$. Thus $\left\{g_{j}(\omega): j=1,2, \ldots, k\right\}$ forms a non-zero orthogonal sequence in $\mathscr{H}$ for almost all $\omega \in \Delta_{0} \cap \Delta_{k}$. Hence

$$
\operatorname{dim} \overline{\overline{\mathscr{K}}}(\omega)=\operatorname{dim} \overline{\operatorname{sp}}\left\{g_{j}(\omega): 1 \leqq j<n+1\right\} \geqq k \quad \mu \text { a.e. on } \Delta \cap \Delta_{k} .
$$

\section{Stationary sequences and their multiplicities}

Let $\mathscr{H}$ and $\mathscr{K}$ be Hilbert spaces. By a stationary sequence we will mean a function $X=\left(X_{n}\right): \mathbf{Z} \rightarrow L(\mathscr{H}, \mathscr{K})$ such that its correlation function $\Gamma(n, m)=X_{m}^{*} X_{n}=\Gamma(n-m, 0)$ depends only on $n-m$. Let $\mathscr{M}(X)=\overline{\mathrm{sp}}\left\{X_{n} x: n \in \mathbf{Z}, x \in \mathscr{H}\right\}$. If $X$ is stationary, then the operator $U: \mathscr{M}(X) \rightarrow \mathscr{M}(X)$ defined by the formula

$$
U\left(\Sigma X_{k} x_{k}\right)=\Sigma X_{k+1} x_{k}, \quad x_{k} \in \mathscr{H},
$$

is unitary. Hence there exists a spectral measure $E(\Delta)$ in $\mathscr{M}(X)$ defined on the Borel $\sigma$-algebra $\mathscr{B}$ in $(-\pi, \pi]$ such that for every $x, y \in \mathscr{M}(X)$

$$
(U x, y)=\int_{-\pi}^{\pi} e^{-i t}(E(d t) x, y) .
$$

The function $\mathscr{F}: \mathscr{B} \rightarrow L^{+}(\mathscr{H})$ defined by the formula $\mathscr{F}(\Delta)=X_{0}^{*} E(\Delta) X_{0}, \Delta \in \mathscr{B}$, is called the spectral measure of the stationary sequence $X=\left(X_{n}\right)$. We will say that the spectral measure of a stationary sequence $X$ is absolutely continuous (or singular) with respect to a nonnegative scalar measure $\mu$ if $(\mathscr{F}(\cdot) x, x) \ll \mu($ or $(\mathscr{F}(\cdot) x, x) \perp \mu)$ for all $x \in \mathscr{H}$. If $\mathscr{H}$ is separable and there exists a function $F_{\mu}(\cdot):(-\pi, \pi] \rightarrow L^{+}(\mathscr{H})$ such that for all $x, y \in \mathscr{H}$ and $\Delta \in \Sigma$,

$$
\int_{\Delta}\left(F_{\mu}(t) x, y\right) \mu(d t)=(\mathscr{F}(\Delta) x, y),
$$

then $F_{\mu}(\cdot)$ is said to be the spectral density of the stationary sequence $\left(X_{n}\right)$ with respect to $\mu$. If $\mu$ is the Lebesgue measure, then we will abbreviate $F_{\mu}(\cdot)$ by $F(\cdot)$ and call $F(\cdot)$ the spectral density of $\left(X_{n}\right)$.

3.2. Definition. Let $\mathscr{H}$ be separable and let $X=\left(X_{n}\right)$ be a stationary $L(\mathscr{H}, \mathscr{K})$-valued sequence. The smallest number $n \in \mathbf{N} \cup\{+\infty\}$ such that there exist $g_{k} \in \mathscr{M}(X), 1 \leqq k<n+1$, with the property $\mathscr{M}(X)=\overline{\mathrm{sp}}\left\{U^{m} g_{k}: 1 \leqq k<n+1, m \in \mathbf{Z}\right\}$ is called the multiplicity of the stationary sequence $X$, and is denoted by $m(X)$.

Note that since $\overline{\mathrm{sp}}\left\{U^{m} x: m \in \mathbf{Z}\right\}=\overline{\mathrm{sp}}\{E(\Delta) x: \Delta \in \mathscr{B}\}$ for every $x \in \mathscr{M}(X)$, $m(X)=m(E, \mathscr{M}(X))$. The spectral types of $E$ in $\mathscr{M}(X)$ will be referred to as the spectral types of the sequence $\left(X_{n}\right)$.

The next theorem gives the description of $m(X)$ in terms of the spectral density of $\left(X_{n}\right)$.

3.3. Theorem. Suppose that $\mathscr{H}$ is separable. Let $F_{\mu}(\cdot)$ be the spectral density of a stationary sequence $X=\left(X_{n}\right) \subset L(\mathscr{H}, \mathscr{K})$ with respect to a $\sigma$-finite nonnegative 
measure $\mu$. Then

$$
m(X)=\mu \text {-ess sup }\left(\operatorname{dim} F_{\mu}(t) \mathscr{H}\right) .
$$

Proof. Consider the stationary $L(\mathscr{H}, \mathscr{W})$-valued sequence $Y=\left(Y_{n}\right)$, where $\mathscr{W}=L^{2}((-\pi, \pi], \mathscr{B}, \mu, \mathscr{H})$, defined by the formula

$$
\left(Y_{n} x\right)(\cdot)=e^{i n} \sqrt{F_{\mu}(\cdot)} x, \quad x \in \mathscr{H} .
$$

Then $m(X)=m(Y)$, since $X$ and $Y$ have the same correlation function. We note that the spectral measure of the shift of the sequence $\left(Y_{n}\right)$ is multiplication by $1_{\Delta}$ in $\mathscr{M}(Y) \subset \mathscr{W}$, which are related by formula (3.1). Using Lemma 2.4 we have $m(X)=m(Y)=\mu$-ess $\sup \left(\operatorname{dim} F_{\mu}(t) \mathscr{H}\right)$.

For every $n \in \mathbf{Z}$ let $\mathscr{M}_{n}(X)=\overline{\mathrm{sp}}\left\{X_{k} x: k \leqq n, x \in \mathscr{H}\right\}$. A stationary sequence $X=\left(X_{n}\right)$ is said to be

(i) deterministic if $\mathscr{M}_{n}(X)=\mathscr{M}(X)$ for all $n \in \mathbf{Z}$,

(ii) regular if $\bigcap_{n \in \mathbf{Z}} \mathscr{M}_{n}(X)=\{0\}$.

3.4. Lemma. If $\mathscr{H}$ is separable and $\left(X_{n}\right)$ is a regular stationary sequence, then $m(X)=\operatorname{dim}\left(\mathscr{M}_{1}(X) \oplus \mathscr{M}_{0}(X)\right)$ and all spectral types of $\left(X_{n}\right)$ are equivalent to the Lebesgue measure.

Proof. Let $n$ be the dimension of $\mathscr{M}_{1}(X) \oplus \mathscr{M}_{0}(X)$ and let $g_{k}, \quad 1 \leqq k<n+1$, be an orthonormal basis in $\mathscr{M}_{1}(X) \oplus \mathscr{M}_{0}(X)$. Then $\mathscr{M}\left(E, g_{k}\right)=\overline{\mathrm{sp}}\left\{U^{m} g_{k}: m \in \mathbf{Z}\right\} \perp$ $\mathscr{M}\left(E, g_{j}\right)$ if $k \neq j$ and $\bigoplus_{k=1}^{n} \mathscr{M}\left(E, g_{k}\right)=\mathscr{M}(X)$, because the process is regular. Since $\left|E(\cdot) g_{k}\right|^{2}=d t / 2 \pi$ for every $k \in \mathbf{N}, 1 \leqq k<n+1$, it follows from Lemma 2.2 (B) that $n=m(E, \mathscr{M}(X))=m(X)$.

For any stationary sequence $\left(X_{n}\right)$ there exists a uniquely determined decomposition of $\left(X_{n}\right)$ into the sum of a deterministic and a regular stationary sequence occurring in the Wold decomposition. On the other hand, every stationary sequence admits a unique decomposition into the sum of two stationary sequences whose spectral measures are absolutely continuous and singular w.r.t. the Lebesgue measure, respectively. Combining these two decompositions we obtain the following three-term decomposition (cf. [1]).

3.5. The or em (Wold Decomposition). Let $X=\left(X_{n}\right)$ be a stationary sequence. There exist stationary sequences $X^{r}=\left(X_{n}^{r}\right), X^{s}=\left(X_{n}^{s}\right)$ and $X^{d}=\left(X_{n}^{d}\right)$ with the properties

(i) $X_{n}=X_{n}^{s}+X_{n}^{d}+X_{n}^{r}, n \in \mathbf{Z}$,

(ii) $\mathscr{M}_{n}(X)=\mathscr{M}_{n}\left(X^{s}\right) \oplus \mathscr{M}_{n}\left(X^{d}\right) \oplus \mathscr{M}_{n}\left(X^{r}\right), n \in \mathbf{Z}$,

(iii) $X^{r}$ is regular,

(iv) $X^{d}$ is deterministic and its spectral measure is absolutely continuous w.r.t. the Lebesgue measure,

(v) $X^{s}$ is deterministic and its spectral measure is singular w.r.t. the Lebesgue measure. 
The decomposition is unique, that is, if $X^{s}, X^{d}$ and $X^{r}$ satisfy (i)-(v), then $X_{n}^{s}=P_{\mathcal{M}^{s}(X)} X_{n}, X_{n}^{d}=P_{\mathscr{M}_{-\infty}(X) \oplus \mathscr{M}^{s}(X)} X_{n}$ and $X_{n}^{r}=P_{\mathscr{M}^{(X) \oplus \mathcal{M}_{-\infty}(X)}} X_{n}$, where $\mathscr{M}_{-\infty}(X)=$ $\bigcap_{n \in \mathbf{Z}} \mathscr{M}_{n}(X), \mathscr{M}^{s}(X)=\left\{x \in \mathscr{M}(X):|E(\cdot) x|^{2} \perp d t\right\}$ and $E$ is the spectral measure in $\mathscr{M}(X)$ associated with $X$, occurring in Formula (3.1).

The next theorem relates multiplicities of the components of $X$ as were described in the Wold Decomposition.

3.6. The or em. Suppose that $\mathscr{H}$ is separable. Let $\left(X_{n}\right)$ be a stationary $L(\mathscr{H}, \mathscr{K})$ valued sequence and let $X_{n}=X_{n}^{s}+X_{n}^{d}+X_{n}^{r}, n \in \mathbf{Z}$, be the Wold Decomposition of $\left(X_{n}\right)$. Then

$$
m(X)=\max \left(m\left(X^{s}\right), m\left(X^{d}\right)+m\left(X^{r}\right)\right) .
$$

Proof. Since the maximal type of $E$ in $\mathscr{M}\left(X^{s}\right)=\mathscr{M}^{s}(X)$ (see Theorem 3.5 for the definition of $\left.\mathscr{M}^{s}(X)\right)$ is singular w.r.t. the maximal type of $E$ in $\mathscr{M}\left(X^{d}+X^{r}\right)$, it follows from Lemma 2.3 (A) that $m(X)=\max \left(m\left(X^{s}\right), m\left(X^{d}+X^{r}\right)\right)$. Since, by Lemma 3.4, all spectral types of $X^{r}$ are equivalent to the Lebesgue measure and since all types of $X^{d}$ are absolutely continuous w.r.t. the Lebesgue measure, we conclude from Lemma $2.3(\mathrm{~B})$ that $m\left(X^{d}+X^{r}\right)=m\left(X^{d}\right)+m\left(X^{r}\right)$.

As an immediate consequence of the formula above we obtain the following generalizations of Theorem 8.3 in [8] and the Wold-Cramér concordance theorem.

3.7. Corollary. If $m(X)=1$ and the spectral measure of the stationary sequence $\left(X_{n}\right)$ is absolutely continuous w.r.t. the Lebesgue measure, then $\left(X_{n}\right)$ is either deterministic or regular.

Proof. Since $m(X)=m\left(X^{d}\right)+m\left(X^{r}\right)=1$, either $X^{d}$ or $X^{r}$ must vanish.

3.8. Corollary. Let $m(X)=1$. If $\left(X_{n}\right)$ is non-deterministic, then the component $\left(X_{n}^{d}\right)$ is absent in the Wold Decomposition, i.e., $X_{n}=X_{n}^{s}+X_{n}^{r}, n \in \mathbf{Z}$.

Proof. Since $X^{r} \neq 0$, we obtain $X^{d}=0$ from the formula in Theorem 3.6 relating the multiplicities of components of $\left(X_{n}\right)$.

In [7] Niemi has observed that the determinism of a stationary sequence can be determined by examining a related family of stationary sequences with multiplicity one. The following theorem contains infinite dimensional versions of Theorems II.1, II.2 and II.3 in [7]. Following [7] let us denote

$$
r(X)=\operatorname{dim}\left(\mathscr{M}_{1}(X) \oplus \mathscr{M}_{0}(X)\right), \quad r(X) \in \mathbf{N} \cup\{+\infty\} .
$$

3.9. Theorem. Suppose that $\mathscr{H}$ is separable. Let $\left(X_{n}\right)$ be a stationary $L(\mathscr{H}, \mathscr{K})-$ valued sequence and let $X_{n}=X_{n}^{s}+X_{n}^{d}+X_{n}^{r}$ be its Wold Decomposition (see Theorem 3.5).

(A) The sequence $\left(X_{n}\right)$ is deterministic if and only if for every $x \in \mathscr{M}(X)$ such that $|E(\cdot) x|^{2} \ll d t$, the sequence $Y_{x, n}=P_{\mathcal{M}(x)} X_{n}, n \in \mathbf{Z}$, is deterministic and $\mathscr{M}(x)=$ $\overline{\mathrm{sp}}\left\{U^{n} x: n \in \mathbf{Z}\right\}$. 
(B) The maximum of the cardinalities of the subsets $\mathscr{A} \subset \mathscr{M}(X)-\{0\}$ with the properties that $\left(Y_{x, n}\right)$ is regular for each $x$ in $\mathscr{A}$ and $\mathscr{M}\left(Y_{x}\right) \perp \mathscr{M}\left(Y_{z}\right)$ for all distinct $x$ and $z$ in $\mathscr{A}$ is equal to $r(X)$.

(C) Let $r(X)<\infty$. If $x_{1}, x_{2}, \ldots, x_{m}$ are nonzero in $\mathscr{M}(X)$ such that $\mathscr{M}\left(Y_{x_{j}}\right) \perp$ $\mathscr{M}\left(Y_{x_{k}}\right)$ for all $k \neq j, 1 \leqq k, j \leqq m$, and all $Y_{x_{k}}, 1 \leqq k \leqq m$, are regular, then $X^{r}=$ $\sum_{k=1}^{m} Y_{x_{k}}$ if and only if $m=r(X)$.

Proof. First note that since $\mathscr{M}(x)$ is invariant under $U$ and $U^{-1}$,

$$
P_{\mathscr{M}(x)} U=U P_{\mathscr{M}(x)} \text { for all } x \in \mathscr{M}(X) .
$$

(A) Since $\mathscr{M}_{n}\left(Y_{x}\right) \supset P_{\mathscr{M}(x)} \mathscr{M}_{n}(X), Y_{x}$ is deterministic for all $x \in \mathscr{M}(X)$ provided $X$ is. Conversely, suppose that $\mathscr{M}_{1}(X) \oplus \mathscr{M}_{0}(X) \neq 0$ and let $x$ be a non-zero element of $\mathscr{M}_{1}(X) \oplus \mathscr{M}_{0}(X)$. Then $x \in \mathscr{M}\left(Y_{x}\right)$ and $\left(x, Y_{x, k} y\right)=\left(x, X_{k} y\right)=0$ for all $k \leqq 0$ and $y \in \mathscr{H}$. Thus $x \in \mathscr{M}_{1}\left(Y_{x}\right) \oplus \mathscr{M}_{0}\left(Y_{x}\right)$ and from $(*) \mathscr{M}\left(Y_{x}\right) \subset \mathscr{M}(x)$. Hence $\left(Y_{x, n}\right)$ is non-deterministic with the multiplicity 1 . Note that by Corollary $3.7\left(Y_{x, n}\right)$ is regular, so $|E(\cdot) x|^{2}$ is equivalent to $d t$.

(B) Let $N$ be the maximum of the cardinalities of the subsets $\mathscr{A} \subset \mathscr{M}(X)$ such that for every $x, z \in \mathscr{A}, x \neq z, \mathscr{M}\left(Y_{x}\right) \perp \mathscr{M}\left(Y_{z}\right)$ and $\left(Y_{x, n}\right)$ is regular for all $x \in \mathscr{A}$. Let $X_{k}, \quad 1 \leqq k<r(X)+1$, be an orthonormal basis in $\mathscr{M}_{1}(X) \oplus \mathscr{M}_{0}(X)$. Then, as the proof of part (A) shows, $x_{k} \in \mathscr{M}_{1}\left(Y_{x_{k}}\right) \oplus \mathscr{M}_{0}\left(Y_{x_{k}}\right)$ and $\left(Y_{x_{k}, n}\right)$ is regular for each $k$. Since $\mathscr{M}\left(x_{k}\right) \perp \mathscr{M}\left(x_{j}\right)$ for $k \neq j$ and $\mathscr{M}\left(x_{k}\right)=\mathscr{M}\left(Y_{x_{k}}\right)$, we have $\mathscr{M}\left(Y_{x_{k}}\right) \perp$ $\mathscr{M}\left(Y_{x_{1}}\right)$ for $k \neq j, 1 \leqq k, j<r(X)+1$. Thus $N \geqq r(X)$.

Conversely, let $x_{k}, 1 \leqq k<N+1$, be a sequence in $\mathscr{M}(X)$ such that all $Y_{x_{k}}$ are regular and $\mathscr{M}\left(Y_{x_{k}}\right) \perp \mathscr{M}\left(Y_{x_{j}}\right)$ for all $k \neq j$. Since $\mathscr{M}_{-\infty}\left(Y_{x_{k}}\right)=\bigcap_{n \in Z} \mathscr{M}_{n}\left(Y_{x_{k}}\right) \supset$ $\cap_{n \in Z} P_{\mathscr{M}\left(x_{k}\right)} \mathscr{M}_{n}(X) \supset P_{\mathscr{M}\left(x_{k}\right)} \mathscr{M}_{-\infty}(X)$ and $Y_{x_{k}}$ are regular, it follows that $\mathscr{M}\left(x_{k}\right) \perp$ $\mathscr{M}_{-\infty}(X)$ for every $1 \leqq k<N+1$. Since $\mathscr{M}\left(x_{k}\right) \supset \mathscr{M}\left(Y_{x_{k}}\right)$ and $\mathscr{M}\left(X^{r}\right)=\mathscr{M}(X) \oplus$ $\mathscr{M}_{-\infty}(X), \mathscr{M}\left(Y_{x_{k}}\right) \subset \mathscr{M}\left(X^{r}\right)$. Since, by Lemma 3.4, the spectral type of each $Y_{x_{k}}$ as well as all the spectral types of $X^{r}$ are equivalent to the Lebesgue measure, we obtain $r(X)=m\left(X^{r}\right) \geqq N$ from Lemma 2.3 (B).

(C) Suppose that $r(X)<\infty$ and let $x_{1}, x_{2}, \ldots, x_{m} \in \mathscr{M}(X)$ be such that for all $1 \leqq k \leqq m \quad Y_{x_{k}}$ are regular and $\mathscr{M}\left(Y_{x_{k}}\right) \perp \mathscr{M}\left(Y_{x_{j}}\right)$ for $k \neq j$. Then, as we have just seen, $\bigoplus_{k=1}^{m} \mathscr{M}\left(Y_{x_{k}}\right) \subset \mathscr{M}\left(X^{r}\right)$ and all spectral types of $Y_{x_{k}}, 1 \leqq k \leqq m$, and $X^{r}$ are equivalent to the Lebesgue measure. Therefore it follows from Lemmas 2.2(B) and 3.4 that $m=r(X)$ if and only if $\bigoplus_{k=1}^{m} \mathscr{M}\left(Y_{x_{k}}\right)=\mathscr{M}\left(X^{r}\right)$, which is equivalent to $X_{n}^{r}=\sum_{k=1}^{m} Y_{x_{k}, n}, n \in \mathbf{Z}$, since $Y_{x_{k}, n}=P_{\mathscr{M}\left(Y_{x_{k}}\right)} X_{n}=P_{\mathscr{M}\left(x_{k}\right)} X_{n}^{r}$.

If $\mathscr{H}$ is separable and a stationary sequence $\left(X_{n}\right)$ has the spectral density $F(\cdot)$ w.r.t. the Lebesgue measure, then, as we will see, it is easy to describe the spectral densities of all $\left(Y_{x, n}\right), x \in \mathscr{M}(X)$. Combining this fact with Theorem 3.8 (A) we obtain the following characterization for the determinism of the sequence $\left(X_{n}\right)$.

3.10. Theorem. Let $\mathscr{H}$ be a separable Hilbert space and let $X=\left(X_{n}\right)$ be a stationary $L(\mathscr{H}, \mathscr{K})$-valued sequence with the spectral density $F(t)$. The sequence 
$\left(X_{n}\right)$ is deterministic if and only if for each weakly measurable function $P(t):(-\pi, \pi] \rightarrow$ $L(\mathscr{H})$ with the properties

(i) $P(t)$ is an orthogonal projection operator dt a.e.,

(ii) $\operatorname{dim}(P(t) \mathscr{H}) \leqq 1$ dt a.e.,

(iii) $P(t) \mathscr{H} \subset \overline{F(t) \mathscr{H}}$ dt a.e.,

the function $F_{p}(t)=\sqrt{F(t)} P(t) \sqrt{F(t)}$ is the spectral density of a deterministic sequence.

Proof. Consider the stationary sequence $Y_{n} \in L\left(\mathscr{H}, L^{2}(\mathscr{H})\right)$ defined by the formula

$$
\left(Y_{n} x\right)(\cdot)=e^{-i n .} \sqrt{F(\cdot)} x, \quad x \in \mathscr{H} .
$$

Since $\left(X_{n}\right)$ and $\left(Y_{n}\right)$ have the same spectral density, they are simultaneously deterministic or non-deterministic. From Theorem 3.9 (A) it follows that the sequence $\left(Y_{n}\right)$ is deterministic if and only if for every $f \in \mathscr{M}(Y)=\left\{g \in L^{2}(\mathscr{H}): g(t) \in \overline{F(t) \mathscr{H}} d t\right.$ a.e. $\}$ (see Lemma 2.4 (ii)) the stationary sequence $Y_{f, n}=P_{\mathcal{M}(f)} Y_{n}, n \in \mathbf{Z}$, is deterministic. We shall prove that a function $G(t)$ is the spectral density of the sequence $Y_{f}=$ $\left(Y_{f, n}\right)$ for some $f \in \mathscr{M}(Y)$ if and only if $G(t)=\sqrt{F(t)} P(t) \sqrt{F(t)} d t$ a.e. for some $L(\mathscr{H})$-valued weakly measurable function $P$ satisfying the conditions (i)-(iii).

Let $f \in \mathscr{M}(Y)$ and let $P(t)=P_{\overline{\mathrm{sp}}\{f(t)\}}, t \in(-\pi, \pi]$. Then for every $g \in \mathscr{M}(Y)$

$$
\left(P_{\mathcal{M}(f)} g\right)(t)=P(t) g(t) d t \quad \text { a.e. }
$$

Thus $\left(Y_{f, n} x\right)(\cdot)=e^{-\mathrm{ir} .} P(\cdot) \sqrt{F(\cdot)} x, x \in \mathscr{H}$, so the spectral density of $\left(Y_{f, n}\right)$ is equal to $\sqrt{F(t)} P(t) \sqrt{F(t)}$ dt. a.e.

Conversely, let $G(t)=\sqrt{F(t)} P(t) \sqrt{F(t)}$, where $P(t)$ is a weakly measurable function satisfying the conditions (i), (ii) and (iii). As we will see in Lemma 4.2, there exists a function $f \in L^{2}(\mathscr{H})$ such that $P(t)=f(t) \otimes f(t) d t$ a.e. Moreover, by (iii), $f \in \mathscr{M}(Y)$. By an analysis similar to the one given in the preceding paragraph we conclude that the spectral density of $\left(Y_{f, n}\right)$ is equal to $G(t)$.

\section{Stationary sequences with multiplicity one}

Theorem 3.10 reduces the problem of determinism of an $L(\mathscr{H}, \mathscr{K})$-valued stationary sequence to that of a family of $L(\mathscr{H}, \mathscr{K})$-valued stationary sequences having spectral densities whose ranges are at most of dimension one. By Theorem 3.3 and Corollary 3.7 each such sequence is either deterministic or regular. In this section we discuss spectral conditions under which a stationary sequence with multiplicity one is regular.

The following assumption remains in force throughout this section. 
4.1. Assumption. Let us assume:

(i) $\mathscr{H}$ is a separable Hilbert space,

(ii) $F(t)$ is a spectral density of a non-zero stationary $L(\mathscr{H}, \mathscr{K})$-valued sequence $X=\left(X_{n}\right)$,

(iii) $\operatorname{dim}(F(t) \mathscr{H}) \leqq 1 d t$ a.e.

Recall that for any $y, z \in \mathscr{H}, y \otimes z$ denotes the one-dimensional linear operator in $\mathscr{H}$ defined by the formula

$$
(y \otimes z) x=(x, z) y .
$$

4.2. Lemma. Under Assumption 4.1 there exists a function $f:(-\pi, \pi] \rightarrow \mathscr{H}$ such that $(x, f) \in L^{2}(\mathbf{C})$ for all $x \in \mathscr{H}$ and

Moreover:

$$
F(t)=f(t) \otimes f(t) d t \quad \text { a.e. }
$$

(a) $|f(t)|^{2}=|F(t)| d t$ a.e.,

(b) $f \in L^{2}(\mathscr{H})$ if and only if $|F(\cdot)| \in L^{1}(\mathbf{C})$,

(c) if $F(t)=f(t) \otimes f(t)=h(t) \otimes h(t)$ dt a.e. and $(x, f(\cdot))$ and $(y, h(\cdot))$ are measurable for all $x, y \in \mathscr{H}$, then there exists a measurable complex-valued function $q(t)$ such that $|q(t)|=1$ dt a.e. and $f(t)=q(t) h(t) d t$ a.e.

Proof. First note that $|F(\cdot)|$ is measurable. Let $\Delta=\{t: F(t) \neq 0\}$ and let $f_{j}(t)=F(t) e_{j}, j=1,2, \ldots$, where $\left\{e_{j}: j=1,2, \ldots\right\}$ is an orthonormal basis in $\mathscr{H}$. Set $g(t)=f_{1}(t)$ if $f_{1}(t) \neq 0, \ldots, g(t)=f_{n}(t)$, if $f_{n}(t) \neq 0$ and $f_{j}(t)=0$ for all $j=1,2, \ldots, n-1, n \in \mathbf{N}$. Let $f(t)=g(t) /|g(t)| \times \sqrt{|F(t)|}$ if $t \in \Delta$ and $f(t)=0$ otherwise. Then $f(t)$ is measurable. Also for every $x \in \mathscr{H}$

$$
(x, f(t)) f(t)=|F(t)|\left(x, \frac{g(t)}{|g(t)|}\right) \frac{g(t)}{|g(t)|}=|F(t)| P_{F(t) \mathscr{t}} x=F(t) x, d t \quad \text { a.e. },
$$

since $F(t)$ is a one-dimensional operator for $t \in \Delta$. Now we proceed with the proof of (a), (b), (c).

(a) $|F(t)|=\sup \{|(F(t) x, x)|:|x| \leqq 1\}=\sup \left\{|(x, f(t))|^{2}:|x| \leqq 1\right\}=|f(t)|^{2} \quad d t$ a.e.

(b) This follows from (a).

(c) Suppose that $F(t)=f(t) \otimes f(t)=h(t) \otimes h(t) d t$ a.e.

Then $\quad \operatorname{sp}\{h(t)\}=\operatorname{sp}\{f(t)\}=F(t) \mathscr{H} \quad$ and $|h(t)|=|f(t)|=\sqrt{|F(t)|} d t \quad$ a.e. Since $\operatorname{dim} F(t) \mathscr{H} \leqq 1 d t$ a.e., there exists a scalar function $q(t)$ such that $q(t) h(t)=$ $f(t)$.

Lemma 4.2 allows the construction of the following model for a stationary sequence whose spectral density satisfies Assumption 4.1. The proof is immediate.

4.3. Lemma. Let $f$ be a measurable $\mathscr{H}$-valued function such that $F(t)=f(t) \otimes$ $f(t) d t$ a.e. and let $Y=\left(Y_{n}\right)$ be the stationary $L\left(\mathscr{H}, L^{2}(\mathrm{C})\right)$-valued sequence defined 
by the formula

$$
\left(Y_{n} x\right)(\cdot)=e^{-i n \cdot}(x, f(\cdot)), \quad x \in \mathscr{H}
$$

Then $F$ is the spectral density of $\left(Y_{n}\right)$.

4.4. Definition. Let $F(\cdot)$ satisfy Assumption 4.1. $F(\cdot)$ is said to be factorable if there exists a measurable $\mathscr{H}$-valued function $a(\cdot)$ such that $(x, a(\cdot)) \in L_{+}^{2}(\mathbf{C})$ for every $x \in \mathscr{H}$, and

$$
F(t)=a(t) \otimes a(t) \quad d t \quad \text { a.e. }
$$

where $L_{+}^{2}(\mathbf{C})$ denotes the subspace of $L^{2}(\mathbf{C})$ consisting of all functions with vanishing negative Fourier coefficients.

The following characterization can be derived from the general theory of factorization (see [5], Theorem 10.3). For completeness we include the proof outlined below.

4.5. Proposition. Under Assumption 4.1 a sequence $\left(X_{n}\right)$ is regular if and only if $F(\cdot)$ is factorable.

Proof. Let $Y=\left(Y_{n}\right)$ be the stationary sequence defined in Lemma 4.3. The sequence $\left(Y_{n}\right)$ is regular if and only if $\mathscr{M}_{0}(Y)=\overline{\operatorname{sp}}\left\{e^{\text {in. }}(x, f(\cdot)): x \in \mathscr{H}, n \geqq 0\right\}$ is not a doubly invariant subspace of $L^{2}(\mathbf{C})$ for the multiplication by $e^{i t}$. An application of the Beurling theorem (e.g. [5], p. 8) completes the proof.

For a finite dimensional Hilbert space $\mathscr{H}$ the characterization of regular stationary sequences with multiplicity one can be found in [8], Theorem 8.2. Unfortunately, this theorem may fail in the infinite dimensional case. The following proposition provides an infinite dimensional version of the result.

4.6. Theorem. Let $\left\{e_{j}: j=1,2, \ldots\right\}$ be an orthonormal basis in $\mathscr{H}$ and let $f_{j k}(t)=\left(F(t) e_{j}, e_{k}\right), k, j=1,2, \ldots$. Then, under Assumption 4.1, the sequence $\left(X_{n}\right)$ is regular if and only if there exists an integer $k$ such that:

(i) $\int_{-\pi}^{\pi} \ln f_{k k}(t) d t>-\infty$,

(ii) there exists a function $\varphi \in L_{+}^{\infty}(\mathbf{C}),|\varphi(t)|=1$ dt a.e., such that for every $j=1,2, \ldots$ the functions

$$
\psi_{j}(t)=\frac{f_{j k}(t)}{f_{k k}(t)} \varphi(t)
$$

are the boundary values of some functions $\tilde{\psi}_{j} \in N_{+}$.

Proof. If $\left(X_{n}\right)$ is regular, then by Proposition $4.5 F(t)=a(t) \otimes a(t)$, where $(x, a(\cdot)) \in L_{+}^{2}(\mathbf{C})$. Let $k$ be such that $\left(e_{k}, a(\cdot)\right) \neq 0$. Then $\left(e_{k}, a(t)\right) \neq 0 d t$ a.e. and $\ln \left|\left(e_{k}, a(\cdot)\right)\right|=\ln f_{k k}(\cdot) / 2$ is in $L^{1}(\mathbf{C})\left([5]\right.$, p. 21). By Theorem 4 in [5], $\left(e_{k}, a(t)\right)=$ $\varphi(t) \eta(t) d t$ a.e., where $\varphi \in L_{+}^{\infty}(\mathbf{C})$ and $\eta$ is an outer function in $L_{+}^{2}(\mathbf{C})$. Thus for 
every $j=1,2, \ldots$

$$
\psi_{j}(t)=\frac{f_{j k}(t)}{f_{k k}(t)} \varphi(t)=\frac{\left(e_{j}, a(t)\right)\left(a(t), e_{k}\right.}{\left(e_{k}, a(t)\right)\left(a(t), e_{k}\right)} \varphi(t)=\frac{\left(e_{j}, a(t)\right)}{\eta(t)}
$$

is the boundary value of a function from $N_{+}$([4], Theorem 2.9).

Conversely, suppose that $F(t)=f(t) \otimes f(t)$ and that $\varphi \in L_{+}^{\infty}(\mathbf{C})|\varphi(t)|=1 d t$ a.e. is such that for every $j \neq k$

$$
\psi_{j}(t)=\frac{f_{j k}(t)}{f_{k k}(t)} \varphi(t)=\frac{\left(e_{j}, f(t)\right)}{\left(e_{k}, f(t)\right)} \varphi(t)=\lim _{r \rightarrow 1} \tilde{\psi}_{j}\left(r e^{i t}\right) d t \quad \text { a.e. },
$$

where $\tilde{\psi}_{j} \in N_{+}$. Since $f_{k k} \in L^{1}(\mathbf{C})$ and $\int_{-\pi}^{\pi} \ln f_{k k}(t) d t>-\infty$, there exists a measurable function $q(t),|q(t)|=1 \quad d t$ a.e. such that $q(t)\left(e_{k}, f(\cdot)\right)$ is an outer function in $L_{+}^{2}$ (C) ([5], p. 21). Let $a(t)=\overline{\varphi(t)} \overline{q(t)} f(t)$. Then $a(\cdot)$ is a measurable $\mathscr{H}$-valued function and for all $j=1,2, \ldots$

$$
\left(e_{j}, a(t)\right)=\left[\frac{\left(e_{j}, f(t)\right)}{\left(e_{k}, f(t)\right)} \varphi(t)\right]\left[q(t)\left(e_{k}, f(t)\right)\right]=\psi_{j}(t)\left[q(t)\left(e_{k}, f(t)\right)\right],
$$

where the first factor $\psi_{j}$ is in $N_{+}$and the second factor $q(\cdot)\left(e_{k}, f(\cdot)\right)$ is in $L_{+}^{2}(\mathbf{C})$. Thus, $\left(e_{j}, a(t)\right)$ is the boundary value of some function from $N_{+}$(see [4] p. 26). Since $\left(e_{j}, a(\cdot)\right) \in L^{2}(\mathbf{C})$, from [4], Theorem 2.11, we have $\left(e_{j}, a(\cdot)\right) \in L_{+}^{2}(\mathbf{C})$ for all $j=1,2, \ldots$. Since $F(t)=a(t) \otimes a(t)$, by Proposition $4.5\left(X_{n}\right)$ is regular.

4.7. Corollary ([8] Theorem 8.2). Suppose that $\operatorname{dim} \mathscr{H}=n<\infty$. Under Assumption 4.1, the sequence $\left(X_{n}\right)$ is regular if and only if there exists $k, 1 \leqq k \leqq n$, such that

(i) $\int_{-\pi}^{\pi} \ln f_{k k}(t) d t>-\infty$,

(ii) the ratios $\delta_{j k}(\cdot)=f_{j k}(\cdot) / f_{k k}(\cdot)$ are the boundary values of functions of the class $N_{\delta}$ for some $\delta>0$ and $j=1,2, \ldots, n$, where $N_{\delta}$ denotes the class of ratios of functions from $H^{\delta}$.

Proof. If $\left(X_{n}\right)$ is regular, then it follows from Proposition 4.5 that there exist a function $a(\cdot)$ and an integer $k, 1 \leqq k \leqq n$, such that $0 \neq\left(e_{k}, a(\cdot)\right) \in L_{+}^{2}(\mathbf{C})$ and such that $\left(e_{j}, a(t)\right) \overline{\left(e_{k}, a(t)\right)}=f_{j k}(t) d t$ a.e. Thus $\delta_{j k}(t)=\left(e_{j}, a(t)\right) /\left(e_{k}, a(t)\right)$ is in $N_{2}$.

Conversely, suppose that $k$ is such that (i) and (ii) hold. Since every function from $H^{\delta}$ can be written in the form $B(z) S(z) H(z)$, where $B$ is a Blaschke product, $S$ is a singular inner function and $H$ is an outer function (see [4], Theorem 2.8), we have

If we take

$$
\delta_{j k}(t)=\lim _{r \rightarrow 1} \frac{B_{j k}\left(r e^{i t}\right) S_{j k}\left(r e^{i t}\right) H_{j k}\left(r e^{i t}\right)}{B_{k k}\left(r e^{i t}\right) S_{k k}} \frac{\left(r e^{i t}\right) H_{k k}\left(r e^{i t}\right)}{.}
$$

$$
\varphi(t)=\lim _{r \rightarrow 1} \prod_{m=1}^{n} B_{m m}\left(r e^{i t}\right) S_{m m}\left(r e^{i t}\right)
$$


for every $j$ the function $\psi_{j}(t)=f_{j k}(t) \varphi(t) / f_{k k}(t)$ is the boundary value of a function in $N_{+}$([4], p. 26). Thus we conclude from Theorem 4.6 that the sequence $\left(X_{n}\right)$ is regular.

The following Lemma is used in Proposition 4.11 and Theorem 4.13. Its proof is given for a more general situation than stated here.

4.8. Lemma. Suppose that $F(\cdot)$ is factorable. If $r(\cdot)$ is a nonnegative function such that

(i) $\int_{-\pi}^{\pi} \ln r(t) d t>-\infty$,

(ii) $r(\cdot)(F(\cdot) x, x) \in L^{1}(\mathbf{C})$ for all $x \in \mathscr{H}$, then $r(t) F(t)$ is factorable.

Proof. Suppose that $F(\cdot)$ is a spectral density, not necessarily of rank one, which can be written in the form $F(t)=A(t)^{*} A(t)$, where $A(\cdot)$ is $L(\mathscr{H})$-valued function such that $A(\cdot) x \in L_{+}^{2}(\mathscr{H})$ for all $x$ in $\mathscr{H}$. Let $r_{1}(t)=\max (r(t), 1), r_{2}(t)=$ $\min (r(t), 1)$. Since

$$
\frac{1}{r_{1}(t)}\left(r_{1}(t) F(t)\right) \leqq F(t) \leqq r_{1}(t) F(t),
$$

it follows from Douglas's theorem ([2], Theorem 1) that there exists a function $A_{1}(t)$ with values in $L(\mathscr{H})$ such that $A_{1}(\cdot) x \in L_{+}^{2}(\mathscr{H})$ for all $x \in \mathscr{H}$ and such that $r_{1}(t) F(t)=A_{1}(t)^{*} A_{1}(t) d t$ a.e. Since $r_{2}(\cdot)$ and $\ln r_{2}(\cdot)$ are in $L^{1}(\mathbf{C}), r_{2}(t)=$ $\overline{\varphi(t)} \varphi(t)$ with $\varphi \in L_{+}^{2}(\mathbf{C})$. Thus $r(t) F(t)=r_{2}(t)\left(r_{1}(t) F(t)\right)=(\varphi(t) A(t))^{*}(\varphi(t) A(t))$, and $\varphi(t) A(t)$ has the desired property.

4.9. Definition ([5], p. 65 and 91). A closed subspace valued function $\mathscr{M}_{t}, \mathscr{M}_{t} \subset \mathscr{H}, t \in(-\pi, \pi]$, is said to be conjugate analytic if there exists a sequence of functions $g_{j} \in L_{-}^{2}(\mathscr{H})$ such that $\mathscr{M}_{t}=\overline{\mathrm{sp}}\left\{g_{j}(t): j=1,2, \ldots\right\} \quad d t$ a.e.

4.10. Remark. If $\operatorname{dim} \mathscr{M}_{t} \leqq 1 d t$ a.e., then $\mathscr{M}_{t}, t \in(-\pi, \pi]$, is conjugate analytic if and only if there exists a function $a(\cdot) \in L_{-}^{2}(\mathscr{H})$ such that $|a(\cdot)|=1 d t$ a.e. and $\mathscr{M}_{t}=\overline{\mathrm{sp}}\{a(t)\} d t$ a.e. or, equivalently, if the orthogonal projection $P_{\mathscr{M}_{t}}$ onto $\mathscr{M}_{t}, t \in(-\pi, \pi]$, is factorable ([5], p. 65).

The next proposition provides a set of sufficient conditions for the factorability of $F(t)$. These conditions involve the conjugate analyticity of the range of $F(t)$ and the behavior of the norm of $F(t)$.

4.11. Proposition. Under Assumption 4.1 the following three conditions are equivalent:

(1) $F(t) \mathscr{H}$ is conjugate analytic and $\int_{-\pi}^{\pi} \ln |F(t)| d t>-\infty$,

(2) $F(t) \mathscr{H}$ is conjugate analytic and there exists $x \in \mathscr{H}$ such that

$$
\ln (F(\cdot) x, x) \in L^{1}(\mathbf{C})
$$


(3) $F(t) \mathscr{H}$ is conjugate analytic and $\ln |F(\cdot)| \in L^{1}(\mathbf{C})$. Moreover, each one of these conditions implies that

(4) $F(t)$ is factorable.

Proof. (1) $\Rightarrow(4)$. This implication is a special case of a more general result (see [2], Theorem 2). We note that the proof could equally be based on Lemma 4.8. In fact, if (1) is satisfied, then $F(t) /|F(t)|=P_{F(t) \mathscr{H}}$ is factorable, so $F(t)=$ $|F(t)| F(t) /|F(t)|$ by Lemma 4.8 .

$(1) \Rightarrow(2)$. Suppose that $F(t)$ satisfies (1). Then it follows from $(1) \Rightarrow(4)$ that $F(t)$ is factorable in the form $F(t)=a(t) \otimes a(t)$, where $(x, a(\cdot)) \in L_{+}^{2}(\mathbf{C})$ for all $x \in \mathscr{H}$. Let $x$ be such that $(x, a(\cdot)) \neq 0 d t$ a.e. Then

$$
\ln (F(\cdot) x, x)=2 \ln |(x, a(\cdot))| \in L^{1}(\mathbf{C}) .
$$

(2) $\Rightarrow(3)$. We note that $P(t)=F(t) /|F(t)|$ is the orthogonal projection onto $F(t) \mathscr{H}$. Since $F(t) \mathscr{H}$ is conjugate analytic, $P(t)$ is factorable (see Remark 4.10). Let $r(t)=|F(t)|$. Then $\int \ln r(t) d t \geqq \int(\ln (F(t) x, x)-2 \ln |x|) d t>-\infty$. Thus by Lemma 4.8, $F(t)=r(t) P(t)$ is factorable. Thus $\ln (F(\cdot) x, x)=\ln r(\cdot)+$ $\ln (P(\cdot) x, x) \in L^{1}(\mathbf{C})$, which implies that $\ln r(\cdot) \in L^{1}(\mathbf{C})$.

(3) $\Rightarrow(1)$. Obvious.

4.12. Remark. Under Assumption 4.1, if $F(t)$ is factorable, then obviously there exists $x \in \mathscr{H}$ such that $\ln (F(\cdot) x, x) \in L^{1}(\mathbf{C})$. Thus the conditions (1), (2), (3), (4) in Proposition 4.11 would be equivalent if the factorability of $F(t)$ implied the conjugate analyticity of its range. If $|F(\cdot)| \in L^{1}(\mathbf{C})$ and $F(t)$ is factorable, then it follows from Lemma 4.2 that $F(t)=a(t) \otimes a(t)$ with $a(\cdot) \in L_{-}^{2}(\mathscr{H})$, so $F(t)$ has a conjugate analytic range. Thus if $|F(\cdot)| \in L^{1}(\mathbf{C})$, then conditions (1), (2), (3), (4) in Proposition 4.11 are equivalent.

Below we prove that, under the Assumption 4.1, the factorability of $F(t)$ implies the range of $F(t)$ to be conjugate analytic if and only if $\ln |F(\cdot)| \in L^{1}(\mathbf{C})$.

4.13. Theorem. Let Assumption 4.1 be satisfied. Suppose that $F(t)$ is factorable. Then $F(t)$ has a conjugate analytic range if and only if $\int_{-\pi}^{\pi} \ln |F(t)| d t<\infty$.

Proof. Suppose that the range of $F(t)$ is conjugate analytic. Since $F(t)$ is factorable, there exists $x \in \mathscr{H}$ such that $\ln (F(\cdot) x, x) \in L^{1}(\mathbf{C})$. From Proposition 4.11 we obtain $\ln |F(\cdot)| \in L^{1}(\mathbf{C})$.

Conversely, suppose that $\int_{-\pi}^{\pi} \ln |F(t)| d t<\infty$ and let $r(t)=1 /|F(t)|$. Since $F(t)$ is factorable, it follows from Lemma 4.8 that the orthogonal projection onto the range of $F(t), P(t)=F(t) /|F(t)|=r(t) F(t)$ is factorable, which in view of Remark 4.10 completes the proof.

4.14. Corollary. Under Assumption 4.1, if $\int_{-\pi}^{\pi} \ln |F(t)| d t<\infty$, then the conditions (1), (2), (3), (4) in Proposition 4.11 are equivalent. 
Theorem 4.13 points to the existence of a factorable spectral density $F(t)$ whose range is not conjugate analytic. This would negate the claim "factorability implies conjugate analyticity" contained in [5], p. 120. Below is an example to this effect.

4.15. Example. Consider the function $g(x)=\exp \left\{\left(1 /\left(1-x^{2}\right)\right)\right\},-1<x<1$. Since $1 /\left(1-x^{2}\right)=\sum_{n=1}^{\infty} x^{2 n}$ and $\exp y=\sum_{n=0}^{\infty} y^{n} / n !$, the function $g(x)$ has the power series representation

$$
g(x)=\sum_{n=0}^{\infty} a_{n} x^{2 n}, \quad-1<x<1,
$$

where $a_{0}=e, a_{n}=\sum_{n=0}^{\infty}(n+k-1) ! / k !(k-1) ! n !, n=1,2, \ldots$. Let

$$
\varphi_{n}(t)=\left[\frac{e^{i t}-e^{-i t}}{2 i}\right]^{n} e^{i n t}, \quad t \in(-\pi, \pi),
$$

and let $r_{n}=a_{n}-\left[a_{n}\right]$, where $\left[a_{n}\right]$ denotes the greatest integer less than or equal to $a_{n}$, $n=0,1,2, \ldots$. Define the sequence $\psi_{0}, \psi_{1}, \ldots$ of analytic polynomials as follows:

$$
\begin{aligned}
& \psi_{0}(t)=a_{0}, \\
& \psi_{1}(t)=\varphi_{1}(t), \ldots, \psi_{\left[a_{1}\right]}(t)=\varphi_{1}(t), \psi_{\left[a_{1}\right]+1}(t)=\sqrt{r_{1}} \varphi_{1}(t) . \\
& \psi_{j}(t)= \begin{cases}\varphi_{k+1}(t), & {\left[a_{1}\right]+\ldots+\left[a_{k}\right]+k+1 \leqq j \leqq\left[a_{1}\right]+\ldots+\left[a_{k+1}\right]+k} \\
\sqrt{r_{k+1}} \varphi_{k}(t), & j=\left[a_{1}\right]+\ldots+\left[a_{k+1}\right]+k+1,\end{cases}
\end{aligned}
$$

for $k \geqq 2$.

Then:

(1) the Fourier coefficients of $\psi_{k}$ vanish outside of $[0,2 k], k=0,1,2, \ldots$,

(2) for every $k=1,2, \ldots, \int_{-\pi}^{\pi}\left|\psi_{k}(t)\right|^{2} d t \leqq 2 \pi$, and

(3) $\sum_{k=0}^{\infty}\left|\psi_{k}(t)\right|^{2}=a_{0}+\sum_{k=1}^{\infty}\left|\psi_{k}(t)\right|^{2}$

$$
\begin{gathered}
=a_{0}+\left(\sum_{j=1}^{\left[a_{1}\right]+1}\left|\psi_{j}(t)\right|^{2}\right)+\sum_{j=\left[a_{1}\right]+2}^{\left[a_{1}\right]\left[a_{2}\right]+2}\left|\psi_{j}(t)\right|^{2}+\ldots=\sum_{k=0}^{\infty} a_{k}|\sin t|^{2 k} \\
=\exp \left\{\frac{1}{1-\sin ^{2} t}\right\}, \quad t \in(-\pi, \pi), \quad t \neq \pm \pi / 2 .
\end{gathered}
$$

Let $\eta_{k}(t)=\psi_{k}(t) e^{i(k+1)^{2} t}, k=0,1,2, \ldots, t \in(-\pi, \pi)$. Then it follows from (1), (2), (3) that $\left\{\eta_{k}: k=0,1,2, \ldots\right\}$ is a sequence of polynomials in $L_{+}^{2}(\mathbf{C})$ such that:

(i) $\int_{-\pi}^{\pi}\left|\eta_{k}(t)\right|^{2} d t \leqq C=2 \pi e$, for every $k=0,1, \ldots$,

(ii) $\int_{-\pi}^{\pi} \eta_{k}(t) \overline{\eta_{j}(t)} d t=0$, for every $k \neq j, k, j=0,1,2, \ldots$,

(iii) $\sum_{k=0}^{\infty}\left|\eta_{k}(t)\right|^{2}=\exp \left\{\frac{1}{1-\sin ^{2} t}\right\}<\infty \quad d t$ a.e.

Let $f(t)$ be the $l^{2}$-valued function defined on $(-\pi, \pi)$ by the formula

$$
f(t)=\left(\overline{\eta_{0}(t)}, \overline{\eta_{1}(t)}, \overline{\eta_{2}(t)}, \ldots\right)
$$


and let $F(t)=f(t) \otimes f(t), t \in(-\pi, \pi)$. Then $F(t)$ is a nonnegative bounded linear operator in $l^{2} d t$ a.e. with the operator norm $|F(t)|=\exp \left\{\left(1 /\left(1-\sin ^{2} t\right)\right)\right\}$. Moreover, for each $x=\left(\alpha_{k}\right) \in l^{2}$ we have

$$
\int_{-\pi}^{\pi}(F(t) x, x) d t=\int_{-\pi}^{\pi}\left|\sum_{k=0}^{\infty} \alpha_{k} \eta_{k}(t)\right|^{2} d t \leqq C \sum_{k=0}^{\infty}\left|\alpha_{k}\right|^{2}=C|x|^{2}<\infty .
$$

Thus $F(t)$ is the spectral density of a stationary sequence with multiplicity 1 . Since for every $x=\left(\alpha_{k}\right) \in l^{2},(x, f(t))=\sum_{n=0}^{\infty} \alpha_{k} \eta_{k}(t) \in L_{+}^{2}(\mathbf{C}), F(t)$ is factorable. However,

$$
\int_{-\pi}^{\pi} \ln |F(t)| d t=\int_{-\pi}^{\pi} \frac{1}{1-\sin ^{2} t} d t=+\infty .
$$

Thus it follows from Theorem 4.13 that the range of $F(t)$ is not conjugate analytic.

\section{References}

[1] Cobanjan, S. A., and A. Weron: Banach space valued stationary processes and their linear prediction. - Dissertationes Math. (Rozprawy Mat.) 125, 1975, 1-45.

[2] Douglas, R. G.: On factoring positive operator functions. - J. Math. Mech. 16, 1966, 119-126.

[3] Dunford, N., and J. T. Schwartz: Linear operators. Part II: Spectral theory. - Interscience Publishers, New York-London, 1963.

[4] Duren, P. L.: Theory of $H^{p}$ spaces. - Academic Press, New York-London, 1970.

[5] Helson, H.: Lectures on invariant subspaces. - Academic Press, New York-London, 1964.

[6] Matveev, R. F.: On multidimensional regular stationary processes. - Theory Probab. Appl. 6, 1961, 149-165.

[7] NIEMI, H.: Subordination, rank and determinism of multivariate stationary sequences. - J. Multivariate Anal. 15: 1, 1984, 99-123.

[8] Rozanov, Yu. A.: Stationary random processes. - Holden-Day. San Francisco-CambridgeLondon-Amsterdam, 1963.

[9] Rozanov, Yu. A.: Innovation processes. - V. H. Winston \& Sons, Washington, D. C., 1977.

Wrocław Technical University

Institute of Mathematics

50-370 Wrocław

Poland

and

Michigan State University

Department of Statistics and Probability

East Lansing, Michigan 48824

USA
Michigan State University

Department of Statistics and Probability East Lansing, Michigan 48824

USA

Received 25 March 1986 